YITP-99-52, KUNS-1597, gr-qc/9909027

\title{
Causal structure of an inflating magnetic monopole
}

\author{
Nobuyuki Sakai* \\ Yukawa Institute for Theoretical Physics, Kyoto University, Kyoto 606-8502, Japan \\ Ken-ichi Nakao \\ Department of Physics, Osaka City University, Osaka 558-8585, Japan \\ Tomohiro Harada \\ Department of Physics, Kyoto University, Kyoto 606-8502, Japan
}

(1 December 1999)

\begin{abstract}
We clarify the causal structure of an inflating magnetic monopole. The spacetime diagram shows explicitly that this model is free from "graceful exit" problem, while the monopole itself undergoes "eternal inflation". We also discuss general nature of inflationary spacetimes.
\end{abstract}

PACS number(s): 04.70.Bw, 14.80.Hv, 98.80.Cq

*Electronic address: sakai@yukawa.kyoto-u.ac.jp 
For the last decade spacetime solutions of magnetic monopoles have been intensively studied in the literature [1 6 . This originated from the rather mathematical interest in static solutions with non-Abelian hair [1]. It was shown [2] that static regular solutions are nonexistent if the vacuum expectation value of the Higgs field $\eta$ is larger than a critical value $\eta_{\mathrm{sta}}$, which is of the order of the Planck mass $m_{\mathrm{Pl}}$.

Linde and Vilenkin independently pointed out that such monopoles could expand exponentially in the context of inflationary cosmology [4]. Because this "topological inflation" model does not require fine-tuning of the initial conditions, it has been attracting attention. In particular, it is recently found that topological inflation takes place in some of the plausible models in particle physics [7].

In Ref. [5] (Paper I), dynamical solutions of magnetic monopoles for $\eta>\eta_{\text {sta }}$ were numerically obtained: monopoles actually inflate if $\eta \gg \eta_{\text {sta }}$. Recently, the causal structure of an inflating magnetic monopole was discussed in Ref. [6]. The spacetime diagrams in Refs. [6.8] showed, for instance, that the inflationary boundary expands along outgoing null geodesics, that is, any observer cannot exit from an inflationary region.

The above argument would be fatal to topological inflation because it implies that reheating never occurs. Therefore, the spacetime structure of topological inflation deserves close examination. In this paper we clarify the causal structure of an inflating magnetic monopole, as a complement of Paper I. As a result, we find that the diagrams in Refs. [6,8] are incorrect, as we shall show below.

In order to see the spacetime structure for numerical solutions, we observe the signs of the expansion of a null geodesic congruence. For a spherically symmetric metric,

$$
d s^{2}=-d t^{2}+A^{2}(t, r) d r^{2}+B^{2}(t, r) r^{2}\left(d \theta^{2}+\sin ^{2} \theta d \varphi^{2}\right),
$$

an outgoing $(+)$ or ingoing $(-)$ null vector is given by $\mathbf{0}$

$$
k_{ \pm}^{\mu}=\left(1, \pm A^{-1}, 0,0\right)
$$

and its expansion $\Theta_{ \pm}$is written as

$$
\Theta_{ \pm}=k_{ \pm ; \theta}^{\theta}+k_{ \pm ; \varphi}^{\varphi}=\frac{2}{B}\left(\frac{\partial B}{\partial t} \pm \frac{1}{A r} \frac{\partial(B r)}{\partial r}\right),
$$

which is defined as the trace of a projection of $k_{; \nu}^{\mu}$ onto a relevant 2-dimensional surface. The derivation of Eq.(3) as well as more general arguments were given by Nakamura et al.

\footnotetext{
${ }^{1}$ In Paper I and Ref. [9], the null vector was expressed as $k_{ \pm}^{\mu}=\left(-1, \pm A^{-1}, 0,0\right)$. The minus sign of $k_{ \pm}^{t}$ was just a typo, and the expression of $\Theta_{ \pm}$, which is the same as Eq.(3), was correct.
} 
[10] We define an "apparent horizon" as the surface with $\Theta^{+}=0$ or $\Theta^{-}=0$ ?. We label those surfaces as S1, S2, etc. in our figures.

In Fig. 1(a), we plot the trajectories of monopole boundaries and of apparent horizons in terms of the proper distance from the center: $X \equiv \int_{0}^{r} A d r^{\prime}$. Here we define the boundary in two ways: $X_{\Phi}$ as the position of $\Phi=\eta / 2$ and $X_{w}$ as the position of $w=1 / 2$, where $\Phi$ and $w$ are the Higgs field and a gauge-field function, respectively. An apparent horizon S1 almost agrees with $X=H_{0}^{-1} \equiv\left[8 \pi V(0) / 3 m_{\mathrm{Pl}}^{2}\right]^{-1 / 2}$, which implies that the monopole core is almost de Sitter spacetime. Figure 1(a) also illustrates that the monopole actually inflates.

Based on the solution in Fig. 1(a), we schematically depict the embedding diagram in Fig. 2. We see that wormhole structure with black hole horizons appears around an inflating core. Because the inflating core becomes causally disconnected from the outer universe, such an isolated region is called a "child universe". The production of child universes were originally discussed by Sato, Sasaki, Kodama, and Maeda (SSKM) [11] in the context of the original inflationary model associated with a first-order phase transition [12]. In monopole inflation, similar spacetime structure appears with a simpler potential (as in Fig. 5) and with natural initial conditions.

In order to see the causal structure of the inflating monopole more closely, we also plot ingoing and outgoing null geodesics in Fig. 1(b). The similar diagram for an inflating global monopole was presented by Cho and Vilenkin [13]. An important feature for the magnetic monopole as well as the global monopole is that the boundary moves inward in terms of the coordinate $r$ and, moreover, it eventually becomes spacelike.

To understand the global structure, which is not completely covered by the numerical solution, we make a reasonable assumption that the core region approaches to de Sitter spacetime and the outside to Reissner-Nordstörom, as in Refs. [3,6]. In each static spacetime, apparent horizons and event horizons are identical; the signs of $\left(\Theta^{+}, \Theta^{-}\right)$are determined as is shown in Fig. 3. The above assumption implies that, although the whole space in the early stage is quite dynamical and an apparent horizon does not coincide with an event horizon, the two horizons later approach to each other. Hence, we can extrapolate the global structure from the structure of apparent horizons in the local numerical solution. From the consistency of the spatial distribution of the signs of $\left(\Theta^{+}, \Theta^{-}\right)$, we conclude that Fig. 3 gives

\footnotetext{
${ }^{2}$ In the literature an "apparent horizon" usually refers to the outermost surface with $\Theta^{+}=0$ in an asymptotically flat spacetime. In this article, however, we call any marginal surface with $\Theta^{+}=0$ or $\Theta^{-}=0$ an apparent horizon.
} 
the only possible embedding.

It is also instructive to compare the inflating monopole solution with SSKM's model of child universe. For SSKM model, Blau et al. studied the details of the boundary motion and the causal structure under the thin-wall approximation [14]. Figure 3 is quite similar to that for their Type E solution, except that the inflationary boundary in SSKM model is lightlike or timelike. The similarity confirms our results in Figs. 1-3, and the difference is also easily understood: the boundary in SSKM model is a domain wall, or a solitonic wave, and hence it cannot be spacelike.

On the other hand, Fig. 4 of Borde, Trodden, and Vachaspati [6] or Fig. 3 of Vachaspati and Trodden [8] are quite different from our fig. 3 in the following respects.

- The inflationary boundary is expressed as outgoing null geodesics (as in Fig. 5(a)).

- The horizon structure is inconsistent with the monopole solution in Fig. 1.

- There is no de Sitter region in the initial time hypersurface.

Their diagrams were not based on any analytic nor numerical solution, and there is no ground that such a spacetime satisfies Einstein equations. Instead they claimed that the horizon argument in Paper I was incorrect for the following reason [6]. $k_{ \pm}^{\mu}$ in Eq.(2) is not the tangent vector associated with affinely parameterized radial null geodesics, and so its expansion $\Theta \equiv k_{: \mu}^{\mu}$ cannot be used to find trapped and anti-trapped surfaces.

The above statement is, however, untrue for the following reason. Their misunderstanding stemmed from different definitions of the expansion $\Theta$. If we adopted the definition $\Theta_{\mathrm{BTV}} \equiv k_{; \mu}^{\mu}=k_{; t}^{t}+k_{; r}^{r}+k_{; \theta}^{\theta}+k_{; \text {varphi }}^{\varphi}$, the null vector $k_{ \pm}^{\mu}$ should be chosen so as to satisfy the geodesic equation. However, it is different from our expression (3), $\Theta=k_{; \theta}^{\theta}+k_{; \varphi}^{\varphi}$. With this expression, it is irrelevant whether $k_{ \pm}^{\mu}$ is a solution of null geodesic equations or not, as we explain as follows. The outgoing $(+)$ or ingoing $(-)$ null vector associated radial null geodesics is generally expressed as $k_{ \pm}^{\mu}=f\left(1, \pm A^{-1}, 0,0\right)$, where $f$ is the positive function which is determined by the null geodesic equations. One can easily check $\Theta_{ \pm}$is simply changed into $f \Theta_{ \pm}$as $k_{ \pm}^{\mu} \rightarrow f k_{ \pm}^{\mu}$. That is, the sign of $\Theta$ can be determined without solving null geodesic equations, as long as one chooses the signs for vector components appropriately. Thus their criticism for Paper I is falsified.

Cosmologically, Fig. 3 tells us that any inflationary region eventually enters a reheating phase because any observer inside the core finally goes out. On the other hand, the monopole boundary continues to expand in terms of the physical size and approaches spatial infinity 
$\left(i^{0}\right)$. Figure 3 thus proves that this model free from "graceful exit" problem, while the monopole itself undergoes "eternal inflation".

Finally, we extend discussions to general nature of inflationary spacetimes. What we have shown is that the boundary of an inflating monopole always goes inward and is spacelike. We should notice here that this behavior is not a special feature of monopole inflation but a general nature of any slow-roll inflation. In any model of slow-roll inflation with a reheating phase, inflation ceases when the inflaton field $\Phi$ becomes larger (or smaller) than a critical value $\Phi_{\mathrm{cr}}$. Therefore, the boundary of an inflationary region is characterized as a hypersurface with $\Phi=\Phi_{\text {cr }}=$ const. Whether a $\Phi=$ const hypersurface is spacelike, timelike or null is up to the sign of $\nabla_{\mu} \Phi \nabla^{\mu} \Phi$. In general spacetimes, the line element is given as

$$
d s^{2}=-\alpha^{2} d t^{2}+\gamma_{i j}\left(d x^{i}+\beta^{i} d t\right)\left(d x^{j}+\beta^{j} d t\right)
$$

where $\alpha$ and $\beta^{i}$ are the lapse function and the shift vector, respectively. Then, $\nabla_{\mu} \Phi \nabla^{\mu} \Phi$ is written as

$$
\nabla_{\mu} \Phi \nabla^{\mu} \Phi=-\frac{1}{\alpha^{2}}\left(\frac{\partial \Phi}{\partial t}\right)^{2}+2 \frac{\beta^{i}}{\alpha^{2}}\left(\frac{\partial \Phi}{\partial t}\right)\left(\frac{\partial \Phi}{\partial x^{i}}\right)+\left(\gamma^{i j}-\frac{\beta^{i} \beta^{j}}{\alpha^{2}}\right)\left(\frac{\partial \Phi}{\partial x^{i}}\right)\left(\frac{\partial \Phi}{\partial x^{j}}\right) .
$$

According to the standard picture of inflation, the scalar field becomes almost homogeneous in a sufficiently inflated region. It follows that there exists a time slicing such that the second and the third terms in the right-hand-side of (5) are negligibly small compared with the first term. Therefore, $\nabla_{\mu} \Phi \nabla^{\mu} \Phi$ is negative in the sufficiently inflated region, which implies that the $\Phi=$ const hypersurface must be spacelike.

Now that we have shown the boundary of an inflating region is spacelike, it is obvious that it moves not outward but inward. Consider, for example, the Higgs potential $V(\Phi)$ as shown in Fig. 4. If the boundary moved outward, any observer would see that the field climbs the potential up from $\Phi \cong \eta$ to $\Phi \cong 0$ and never rolls down to $\Phi \cong \eta$; however, such classical dynamics is improbable. We therefore conclude that the causal structure of an inflating local region is schematically described by Fig. 5(b). Although one may intuitively draw a diagram like Fig. 5(a), or Fig. 2 of Vachaspati and Trodden [8], it is incorrect.

\section{ACKNOWLEDGMENTS}

The numerical computations of this work were carried out at the Yukawa Institute Computer Facility. N.S. and T.H. were supported by JSPS Research Fellowships for Young Scientists, No. 9702603 and No. 9809204. 


\section{REFERENCES}

[1] For a review of spacetime solutions with non-Abelian fields, see, e.g., M.S. Volkov and D.V. Gal'tsov, preprint hep-th/9810070.

[2] M.E. Ortiz, Phys. Rev. D 45, R2586 (1992); K. Lee, V.P. Nair, and E.J. Weinberg, ibid. 45, 2751 (1992); P. Breitenlohner, P. Forgàcs, and D. Maison, Nucl. Phys. B383, 357 (1992); ibid. B442, 126 (1995).

[3] T. Tachizawa, K. Maeda, and T. Torii, Phys. Rev. D 51, 4054 (1995).

[4] A. Linde, Phys. Lett. B 327, 208 (1994); A. Vilenkin, Phys. Rev. Lett. 72, 3137 (1994).

[5] N. Sakai, Phys. Rev. D 541548 (1996).

[6] A. Borde, M. Trodden, and T. Vachaspati, Phys. Rev. D 59, 43513 (1998).

[7] J. Ellis, N. Kaloper, K.A. Olive, and J. Yokoyama, Phys. Rev. D 59, 103503 (1999); Izawa K.-I., M. Kawasaki, and T. Yanagida, Prog. Theor. Phys. 101, 1129 (1999); N. Sakai and J. Yokoyama, Phys. Lett. B 456, 113 (1999).

[8] T. Vachaspati and M. Trodden, Phys. Rev. D 61, 023502 (1999).

[9] N. Sakai, H. Shinkai, T. Tachizawa, and K. Maeda, Phys. Rev. D 53, 655 (1996).

[10] T. Nakamura, K. Oohara, and Y. Kojima, Prog. Theor. Phys. Suppl. 90, 1 (1987).

[11] K. Sato, M. Sasaki, H. Kodama, and K. Maeda, Prog. Theor. Phys. 65, 1443 (1981); H. Kodama, M. Sasaki, K. Sato, and K. Maeda, ibid. 66, 2052 (1981); H. Kodama, M. Sasaki, and K. Sato, ibid. 68, 1979 (1981); K. Maeda, K. Sato, M. Sasaki, and H. Kodama, Phys. Lett. 108B, 98 (1982). K. Sato, H. Kodama, M. Sasaki, and K. Maeda, ibid. 108B, 103 (1982).

[12] K. Sato, Mon. Not. Roy. Astron. Soc. 195, 467 (1981); Phys. Lett. 99B, 66 (1981); A.H. Guth, Phys. Rev. D 23, 347 (1981).

[13] I. Cho and A. Vilenkin, Phys. Rev. D 56, 7621 (1997).

[14] S. Blau, E. Guendelman, and A. Guth, Phys. Rev. D 35, 1747 (1987). 


\section{FIGURES}

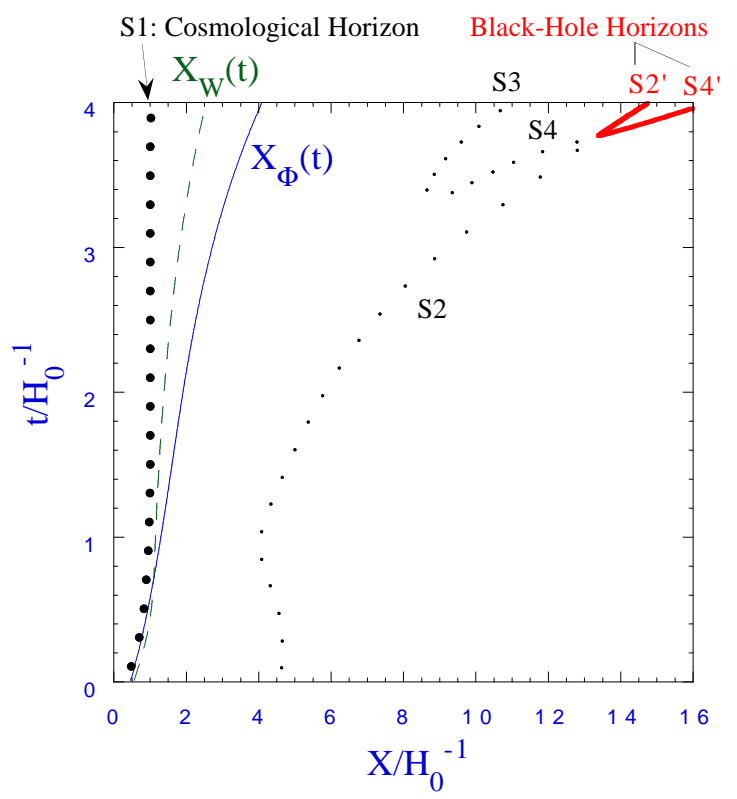

(a)

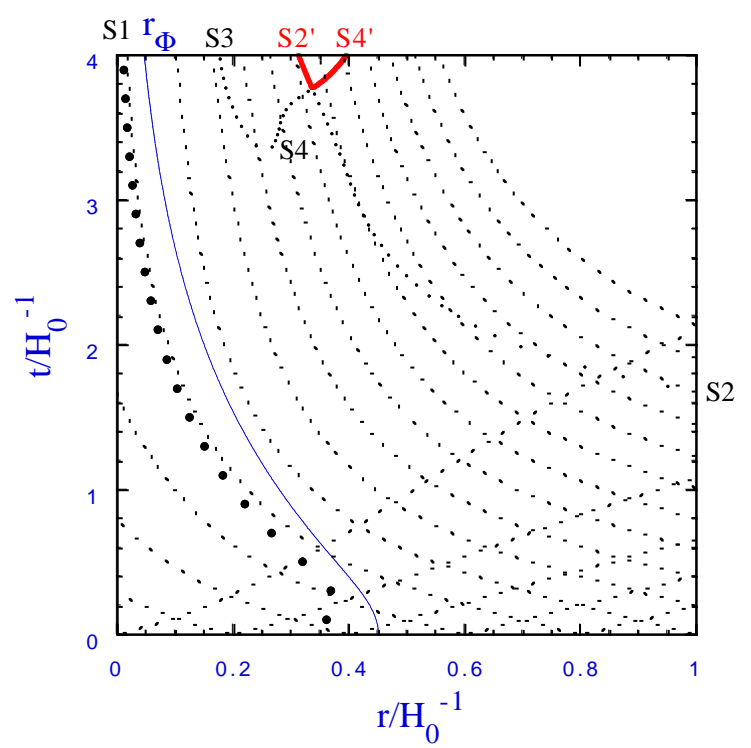

(b)

FIG. 1. A solution of an inflating magnetic monopole. (a) is the reproduction of Fig. 4(a) of Paper I: we plot the trajectories of monopole boundaries and of apparent horizons in terms of the proper distance from the center, $X$; dotted lines denote apparent horizons; we normalize time and length by the horizon scale defined as $H_{0}^{-1} \equiv\left[8 \pi V(0) / 3 m_{\mathrm{Pl}}^{2}\right]^{-1 / 2}$. In (b) we plot ingoing and outgoing null geodesics (dotted lines) besides the above-mentioned trajectories in a $t-r$ diagram; the boundary moves inward and eventually becomes spacelike. 


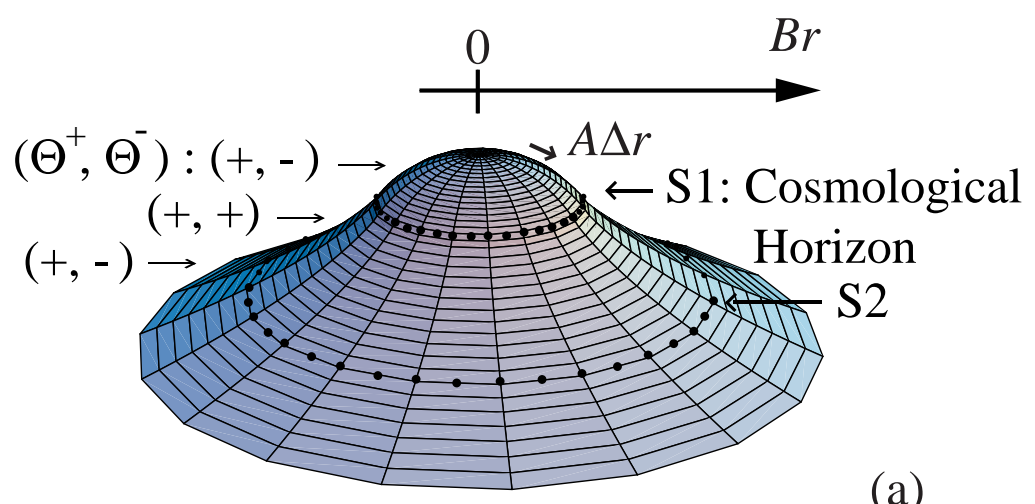

(a)

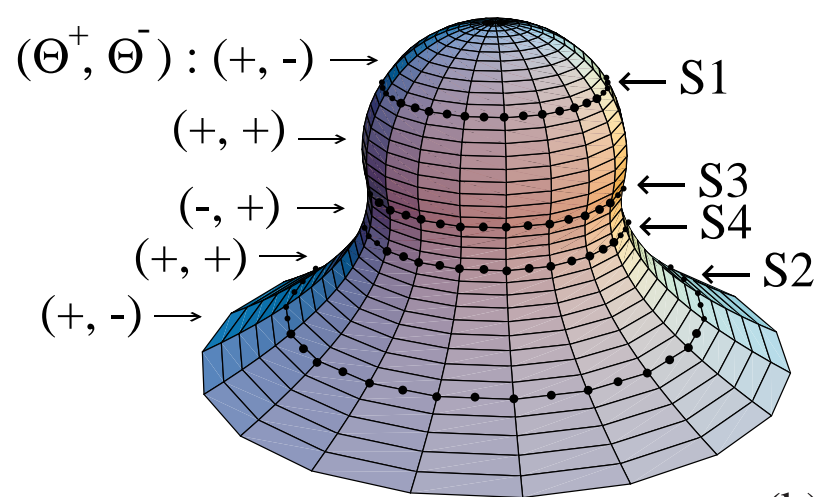

(b)

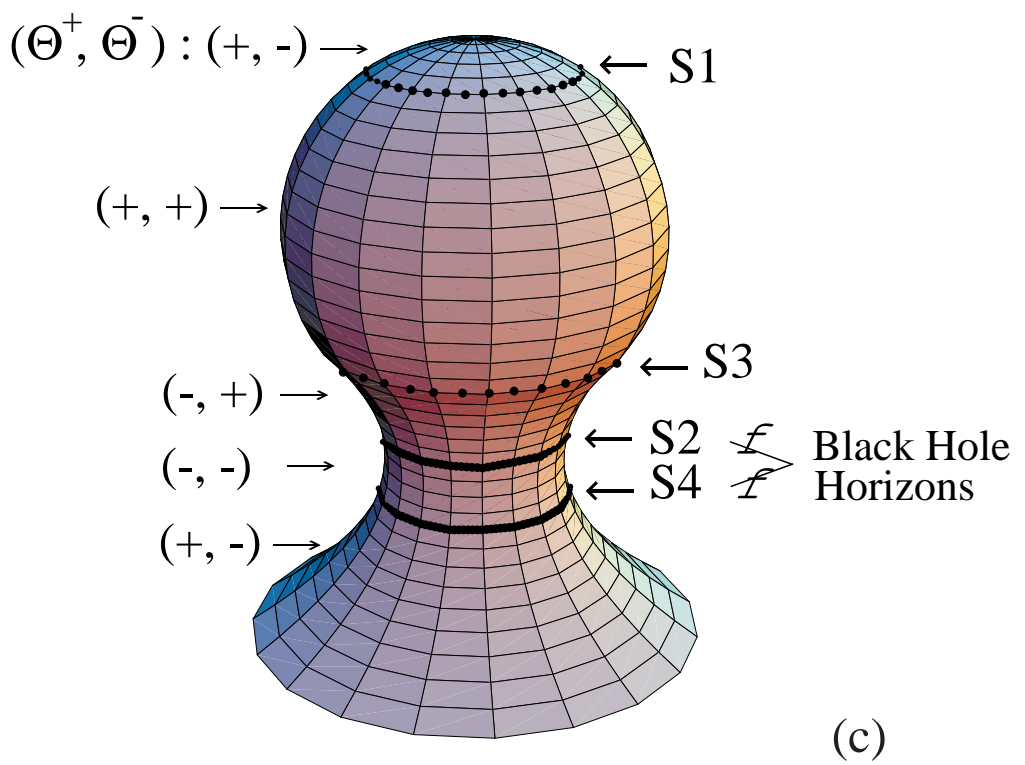

FIG. 2. Schematic embedding diagram of the solution in Fig. 1. 

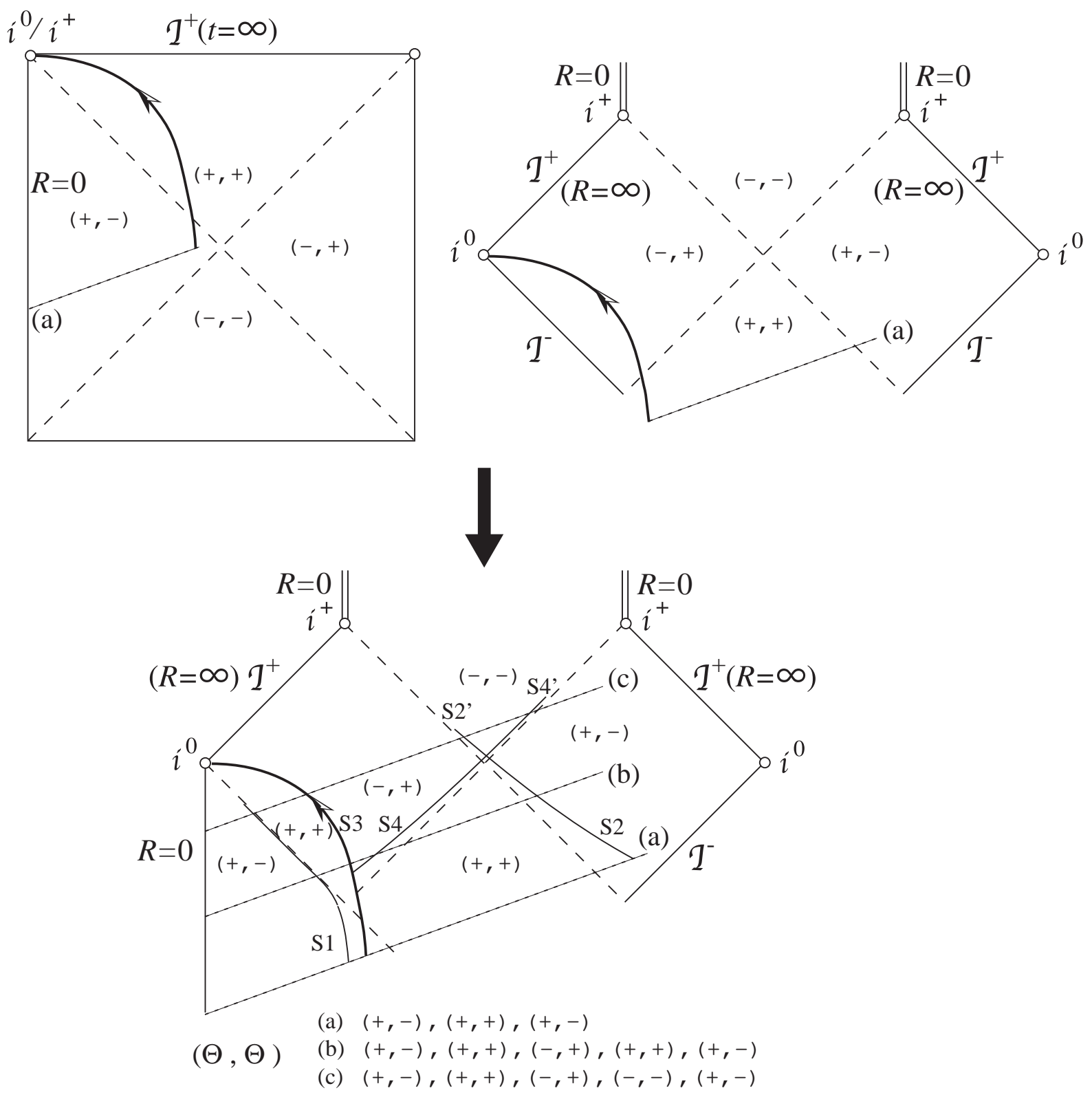

FIG. 3. Possible conformal diagram for the spacetime of an inflating magnetic monopole. The core region is approximated to be de Sitter spacetime and the outside to be ReissnerNordstörom. The upper figure shows how the monopole boundary is embedded in each spacetime separately, and the lower figure shows a complete spacetime. $\mathcal{I}^{+}$and $\mathcal{I}^{-}$represent future and past null infinity, $i^{+}$represents future timelike infinity, and $i^{0}$ represents spacelike infinity. Long-dashed lines denote event horizons, which are identified with apparent horizons in de Sitter or Reissner-Nordstörom spacetime. Short-dashed lines (a), (b), and (c) denote time-slices, which correspond to the embedding diagrams (a), (b), and (c) of Fig. 2, respectively. For reference, we schematically depict the trajectories of apparent horizons. We also write down the signs of the expansion $\Theta$. The structure of apparent horizons, which is determined by the sings of $\Theta^{ \pm}$, completely agrees with that in Fig. 2. 


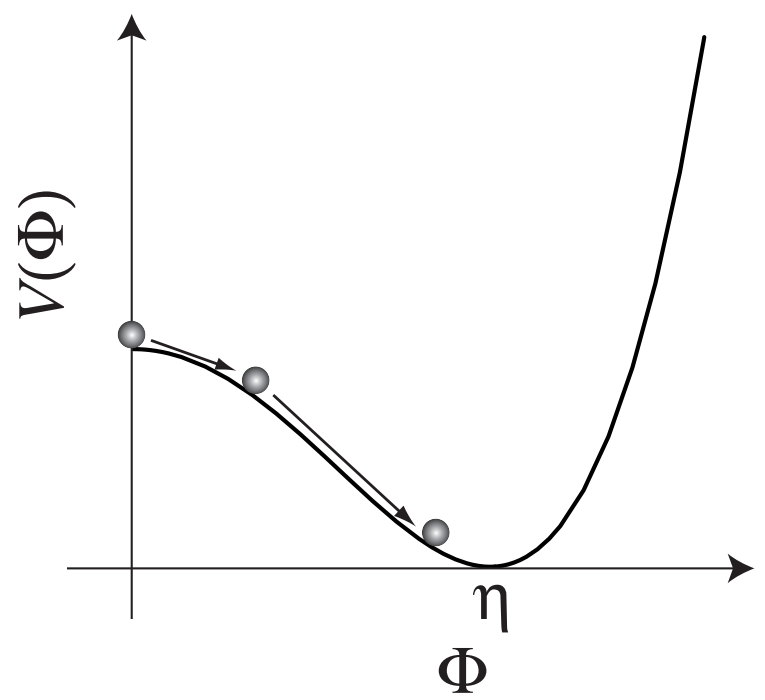

FIG. 4. Higgs potential which induces inflation. Any observer in the inflationary phase sees that the field rolls down from $\Phi \cong 0$ to $\Phi \cong \eta$.

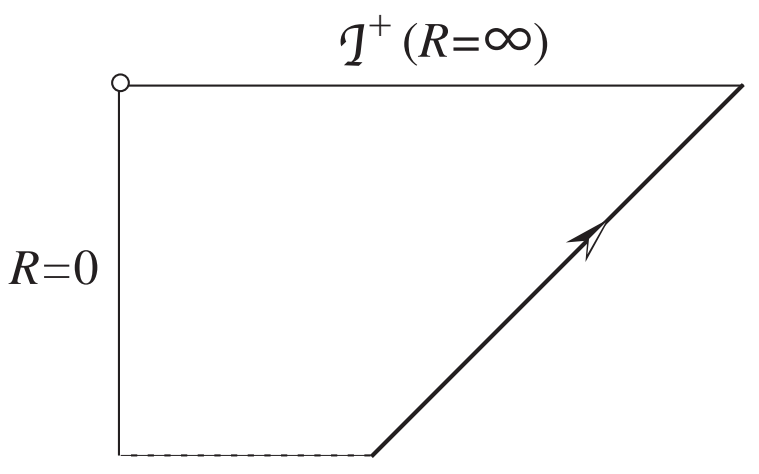

(a) (incorrect)

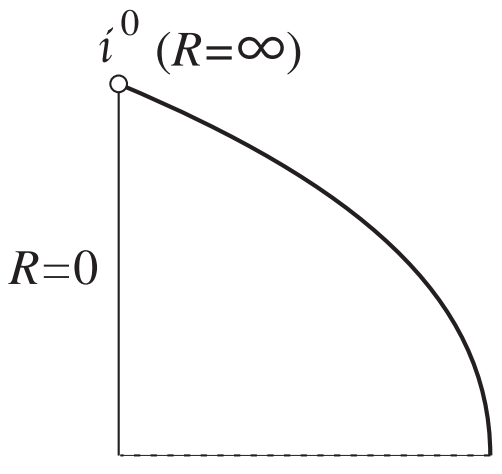

(b)

FIG. 5. Schematic conformal diagram for general inflationary spacetimes. A short-dashed line denotes an initial hypersurface. Because an inflationary boundary moves inward and becomes spacelike, it is described by not (a) but (b). 